Arquivos Brasileiros de Educação Física

Brazilian Archives of Physical Education

ABEF

\title{
Em qual região do Brasil, os corredores apresentam o melhor desempenho?
}

\section{In which Brazilian regions, road runners have the best performance?}

\section{En qué región de Brasil presentan los corredores el mejor rendimiento?}

\author{
Mabliny Thuany \\ Thayse Natacha Gomes
}

\section{Resumo}

O crescimento no número de praticantes de corrida de rua tem sido evidenciado ao longo das últimas décadas, assim como o número de eventos da modalidade, e a mobilidade de corredores pelo país para participar destes eventos. A proposta do presente estudo foi verificar se há diferenças no desempenho de corredores de rua brasileiros, consoante região de residência, bem como investigar se a região pode ser considerada um preditor de performance em corredores de ambos os sexos. A amostra foi composta por 1158 corredores de rua, de ambos os sexos (39,6\% mulheres; $60,4 \%$ homens), oriundos de todas as regiões do país. A obtenção dos dados ocorreu a partir do envio de um questionário online, onde foram obtidas informações a respeito do sexo, idade, massa corporal $(\mathrm{kg})$, altura $(\mathrm{m})$, volume de corrida/semana $(\mathrm{km})$, ritmo de corrida $(\mathrm{seg} / \mathrm{km})$ e região de residência. Teste Kruskal-Wallis $(\mathrm{H})$, seguido do teste U-man Whitney (U), foram computados para identificar diferenças no ritmo de corrida entre as regiões brasileiras, consoante sexo. Em seguida, realizou-se análise de regressão robusta, com ajuste para a idade, para verificar associação entre a região de residência com a performance, tendo a região Sul como referência, considerando p<0,05. Verificaram-se diferenças significativas na média do ritmo de corrida entre as mulheres das cinco regiões $(\mathrm{H}=25,52 ; \mathrm{p}<0,001)$, sendo que aquelas oriundas da região Sul apresentaram melhor desempenho do que seus pares das regiões Sudeste $(\mathbf{U}=60,9 ; \mathrm{p}=0,016)$, Norte $(\mathbf{U}=26,7 ; \mathrm{p}=0,027)$, Centro-Oeste $(\mathbf{U}=25,3 ; \mathrm{p}=0,004)$, Nordeste $(\mathbf{U}=93,9 ; \mathrm{p}<0,001)$. Exceção para a região Norte, corredoras residentes em outras regiões do país experimentam redução da performance comparativamente às da região Sul. A maior redução na performance foi verificada para atletas residentes na região centro-oeste $(\square=49,1$; IC95\%=20,3 - 77,8). Para o sexo masculino, verificou-se associação significativa e inversa entre atletas residentes na região Nordeste e o desempenho, com aumento de $\approx 17$ segundos por quilômetro $(\square=17,1$; IC95\%=2,9 - 31,4). Corredores de rua residentes na região Sul do país apresentaram performance superior a seus pares, para ambos os sexos. Tais resultados podem reforçar a importância da realização de eventos e existência de apoio à modalidade nas demais regiões.

Palavras-chave: Corrida de rua. Performance. Endurance.

\footnotetext{
Abstract

The increase in the number of running practitioners have being observed in last decades, as well as the number of running events, and the runners' travelling around the country to take part in these events. The aim of the present study was to identify differences in performance among Brazilian road runners, by region of residence, as well as to investigate if the regions can be considered as predictor of runners' performance of both sexes. The sample comprised 1158 road runners, of both sexes

1. Centro de Investigação, Formação, Inovação e Intervenção em Desporto - CIFI2D, Faculdade de Desporto, Universidade do Porto - Porto, Portugal. Programa de Pós-Graduação em Educação Física (PPGEF/UFS). 2. Departamento de Educação Física Universidade Federal de Sergipe, São Cristóvão/SE - Brasil. E-mail para correspondência: mablinysantos@gmail.com . Este conteúdo utiliza a Licença Creative Commons Attribution 4.0 International License Open Access. This content is licensed under a Creative Commons attribution-type BY ISSN 2595 - 0096.
} 
(39.6\% women; 60.4\% men), from all Brazilian regions. Data were collected through an online questionnaire, which provided information related to sex, age, body mass $(\mathrm{kg})$, height $(\mathrm{m})$, running volume/week $(\mathrm{km})$, running pace $(\mathrm{seg} / \mathrm{km})$, and region of residence. The Kruskal-Wallis test $(\mathrm{H})$, followed by the U-Man Whitney test (U), were computed to identify differences in the running pace between the Brazilian regions, by sex. Following, age-adjusted robust regression analyses was computed, to estimate the association between the region of residence and the performance, considering the South region as the reference one, with $\mathrm{p}<0.05$. Statistically significant differences in the mean running pace were observed between women from the five regions $(\mathrm{H}=25.52 ; \mathrm{p}<0.001)$, where those from the South region showed better performance than their peers from the Southeast $(\mathrm{U}=60.9 ; \mathrm{p}=0.016)$, North $(\mathrm{U}=26.7 ; \mathrm{p}=0.027)$, Midwest $(\mathrm{U}=25.3 ; \mathrm{p}=0.004)$, and Northeast $(\mathrm{U}=93.9$; $\mathrm{p}<0.001)$ regions. Except for the North region, female runners from the other Brazilian regions showed a decrease in performance when comparing against their peers from the South region. The highest reduction in the performance was observed in those athletes from the Midwest region ( $\square$ $=49.1$; IC95\%=20.3 - 77.8). Among male runners, it was found an inverse and significant association between athletes from the Northeast region and the performance, with an increase of $\approx 17$ seconds per kilometre $(\square=17.1$; IC95\%= 2.9 - 31.4). Road runners from the Brazilian South region showed the best performance, in both sexes. These results reinforce the relevance of the promotion of events and the supporting of the modality in all the regions.

Keywords: Running. Performance. Endurance.

\section{Resumen}

El crecimiento en el número de corredores callejeros se ha evidenciado en las últimas décadas, así como el número de eventos en el deporte y la movilidad de corredores en todo el país para participar en estos eventos. El propósito de este estudio fue verificar si existen diferencias en el desempeño de los corredores callejeros brasileños, según la región de residencia, así como investigar si la región puede considerarse un predictor del desempeño en corredores de ambos sexos. La muestra estuvo compuesta por 1158 corredores callejeros, de ambos sexos (39,6\% mujeres; 60,4\% hombres), de todas las regiones del país. La recolección de datos se realizó mediante el envío de un cuestionario en línea, donde se obtuvo información sobre sexo, edad, masa corporal $(\mathrm{kg})$, altura $(\mathrm{m})$, volumen de carrera / semana $(\mathrm{km})$, ritmo de carrera $(\mathrm{seg} / \mathrm{km})$ y región de residencia. La prueba de KruskalWallis (H), seguida de la prueba de U-man Whitney (U), se calcularon para identificar las diferencias en el ritmo de carrera entre las regiones brasileñas, según el género. Luego, se realizó un análisis de regresión robusto, con ajuste por edad, para verificar la asociación entre la región de residencia y desempeño, con la región sur como referencia, considerando $\mathrm{p}<0.05$. Hubo diferencias significativas en el ritmo de carrera promedio entre las mujeres de las cinco regiones $(\mathrm{H}=25.52 ; \mathrm{p}<0.001)$, y las de la región Sur se desempeñaron mejor que sus pares del Sudeste $(\mathrm{U}=60.9 ; \mathrm{p}=0.016)$, Norte (U $=26,7 ; \mathrm{p}=0,027)$, Medio Oeste $(\mathrm{U}=25,3 ; \mathrm{p}=0,004)$, Noreste $(\mathrm{U}=93,9 ; \mathrm{p}<0,001)$. A excepción de la región Norte, los corredores que residen en otras regiones del país experimentan un rendimiento reducido en comparación con la región Sur. La mayor reducción en el rendimiento se verificó para los atletas que residen en la región Medio Oeste $(\square=49,1 ; \mathrm{IC} 95 \%=20,3$ - 77,8). Para los hombres, hubo una asociación significativa e inversa entre los atletas que viven en la región Noreste y el rendimiento, con un aumento de $\approx 17$ segundos por kilómetro $(\square=17,1$; IC del $95 \%=$ 2,9 - 31,4). Los corredores callejeros que residen en la región sur del país se desempeñaron mejor que sus pares, para ambos sexos. Estos resultados pueden reforzar la importancia de la realización de eventos y la existencia de apoyo al deporte en otras regiones.

Palabras clave: Carrera. Rendimiento. Resistencia Física. 


\section{INTRODUÇÃO}

O crescimento no número de praticantes de corrida de rua, a nível mundial, tem sido evidenciado ao longo das últimas décadas, tendo alcançado o pico no ano de 2016, seguido de uma ligeira redução'. No Brasil, resultados do relatório de "Práticas de Esporte e Atividade Física”, indicam que $\approx 24,6 \%$ dos brasileiros praticam corrida/caminhada frequentemente ${ }^{2}$. Adicionalmente, nos estados de São Paulo e Rio de Janeiro, verificou-se um incremento de mais de $90 \%$ no número de eventos de corrida de rua durante os anos de 2007 a $2015^{3}$. No ano de 2019, esse crescimento pôde ser vislumbrado pela registro de aproximadamente 600 provas de corrida de rua, alocadas em diferentes estados do país ${ }^{4}$.

Entretanto, o incremento observado no número de eventos não ocorre de modo equitativo em todo o país. Por exemplo, as regiões Sudeste e Sul concentram grande parte dos eventos, bem como as mais tradicionais e principais provas do calendário anual, como a corrida de São Silvestre, Volta internacional da Pampulha, Maratona de São Paulo, Maratona de Florianópolis, Meia Maratona do Rio de Janeiro e Maratona de Porto Alegre ${ }^{5}$. Tal fato corrobora para a ocorrência de um fenômeno descrito previamente como OUTrun, que se caracteriza pela grande saída de corredores dos estados localizados nas regiões Norte, Nordeste e Centro-oeste para participação em eventos realizados em outras localidades, sobretudo nas regiões Sul e Sudeste ${ }^{6}$. Daqui que estudos prévios tenham indicado a associação entre fatores econômicos e a participação na modalidade ${ }^{7}$, ilustrado, sobretudo, pela participação em eventos realizados em outros estados ${ }^{6}$.

No contexto de alto rendimento, estudo realizado previamente indicou que as regiões Sudeste e Sul são aquelas responsáveis pelo maior número de atletas de alto rendimento do país ${ }^{8,9}$. Esses resultados foram associados a características socioeconômicas das referidas regiões, similarmente ao observado em estudos envolvendo diferentes modalidades $^{10,11}$ e programas esportivos ${ }^{12}$. Deste modo, a proposta do presente estudo é verificar se há diferenças no desempenho de corredores de rua brasileiros, consoante região de residência, bem como se a região pode ser considerada um preditor de performance em corredores de ambos os sexos. Com base em estudos prévios, que evidenciam um maior quantitativo de corredores das regiões Sudeste e Sul no tocante à participação em eventos da modalidade, bem como evidências que sugerem que os corredores de elite destas regiões tendem a figurar entre aqueles com melhor desempenho a nível nacional, a que se associam melhor infraestrutura para 
treinamento e número de eventos de corrida de rua sediados ${ }^{8,9,12,13}$, hipotetizamos que no contexto recreacional, as referidas regiões também serão aquelas em que os participantes apresentam o melhor desempenho.

\section{METODOLOGIA}

\section{Design e amostra}

$\mathrm{O}$ trabalho apresenta um design transversal, realizado com praticantes de corrida de rua brasileiros, oriundos do projeto In Track ${ }^{14}$. Foram considerados como critérios de inclusão: ser praticante de corrida de rua e responder ao questionário eletrônico utilizado na pesquisa. Indivíduos com idade inferior aos 18 anos, ou que não informassem o estado de residência, bem como informações referentes ao desempenho na corrida de rua, não foram considerados nas análises. A amostra foi composta por 1158 corredores de rua, de ambos os sexos (39,6\% mulheres; $60,4 \%$ homens), oriundos de todas as regiões do país (Centro-oeste: 8,6\%; Nordeste: 36,3\%; Norte: 6,8\%; Sudeste: 36,4\% e Sul: 11,8\%). Todos os corredores foram informados a respeito dos propósitos do estudo. A pesquisa foi aprovada pelo Comitê de Ética em Pesquisa da Universidade Federal de Sergipe (protocolo $\left.\mathrm{n}^{\mathrm{o}} 3.558 .630\right)$.

\section{Procedimentos para coleta dos dados}

A obtenção dos dados ocorreu a partir do envio de um questionário online, denominado "Caracterização do perfil e fatores associados ao desempenho em corredores de rua" ${ }^{15}$. O instrumento foi desenvolvido e validado com a finalidade de permitir a obtenção de informações referentes a um conjunto de domínios, como por exemplo: identificação do corredor; variáveis antropométricas; perfil sociodemográfico; percepção sobre a influência do ambiente na prática e características do treinamento. Para os propósitos do presente trabalho, foram utilizadas informações referentes ao sexo, idade, massa corporal $(\mathrm{kg})$, altura $(\mathrm{m})$, volume de corrida/semana $(\mathrm{km})$, ritmo de corrida $(\mathrm{seg} / \mathrm{km})$ e região de residência. O índice de massa corporal (IMC - $\mathrm{kg} / \mathrm{m}^{2}$ ) foi calculado, com base em fórmula estandardizada.

Análise estatística

Informações descritivas foram apresentadas em média (desvio padrão) e frequências (\%). A normalidade dos dados foi testada para o ritmo de corrida através do teste de Kolmogorov-Sminorv, considerando-se as regiões. O teste Kruskal-Wallis $(\mathrm{H})$, seguido do teste U-man Whitney (U), foram realizados para identificar diferenças no ritmo de corrida entre as regiões brasileiras, divididos por sexo. Em seguida, análise de regressão linear robusta, com ajuste para a idade, foi utilizada para verificar se a região de residência pode ser considerada um preditor da performance. Para isso, a variável região foi recodifica em dummies, e a região Sul foi considerada como 
categoria de referência, para ambos os sexos, visto que apresenta os melhores valores para a variável dependente. Todas as análises foram realizadas no software SPSS 26, adotando-se 95\% de confiança.

\section{RESULTADOS}

A média de idade, IMC e volume de corrida/semana foram 38,4 $\pm 18,7$ anos, 23,8 $\mathrm{kg} / \mathrm{m}^{2}$ e $23 \mathrm{~km}$ para mulheres e $37,6 \pm 9,9$ anos, $24,8 \mathrm{~kg} / \mathrm{m}^{2}$ e $48 \mathrm{~km}$ para homens, respectivamente. Informações descritivas, consoante região de residência, podem ser verificadas na tabela 1 . Os participantes oriundos da região Sul apresentam os menores valores de IMC $(23,9 \pm 3,2)$ e ritmo de corrida $(312,6 \pm 57,2)$.

Diferenças significativas para o desempenho consoante região foram verificadas para o sexo feminino $(\mathrm{H}=25,52$; $\mathrm{p}$ $<0,001)$, onde corredoras oriundas da região Sul apresentaram melhor desempenho, comparativamente a seus pares da região Sudeste $(\mathrm{U}=60,9 ; \mathrm{p}=0,016)$, Norte $(\mathrm{U}=26,7$; $\mathrm{p}=0,027)$, Centro-Oeste $(\mathrm{U}=25,3 ; \mathrm{p}=0,004)$ e Nordeste $\quad(\mathrm{U}=93,9 ; \quad \mathrm{p}<0,001)$. Entre os homens, não foram observadas diferenças significativas (figura 1).

Os resultados da regressão robusta são apresentados nas tabelas 2 e 3, para mulheres e homens, respectivamente. Quando ajustados para a idade dos corredores verificase que, exceto para a região Norte, mulheres residentes nas demais regiões do país experimentam redução da performance comparativamente às atletas da região Sul. A maior redução na performance pode ser observada para aquelas residentes na região centro-oeste $(B=49,1 ;$ IC95\%= 20,3 - 77,8). Para o sexo masculino, verifica-se uma associação significativa entre atletas residentes na região Nordeste com o desempenho, indicando uma relação inversa com a performance, com aumento de $\approx 17$ segundos a cada quilômetro, comparativamente a seus pares da região Sul $(B=17,1$; IC95\%=2,9 $31,4)$.

\section{DISCUSSÃO}

Os últimos anos foram marcados por um crescimento no número de eventos de corrida de rua a nível nacional ${ }^{3}$, figurando-se em um setor que movimenta a economia e o turismo no país ${ }^{16}$. Nesse sentido, é comum que os corredores se desloquem entre os estados/regiões, para participação em eventos. A proposta do presente trabalho foi verificar se há diferenças no desempenho de corredores brasileiros, consoante região de residência. Com base em dados que indicam maior porcentagem de atletas de alto rendimento oriundos da região Sudeste e Sul do país ${ }^{17,18}$, bem como melhor infraestrutura para treinamento ${ }^{8}$ e maior número de eventos de corrida de rua sediados, foi hipotetizado 
que, no âmbito recreacional, os corredores residentes nas referidas regiões também apresentariam a melhor performance.

Não obstante os corredores do sexo masculino residentes na região Sul apresentarem valores mais baixos para o ritmo de corrida comparativamente às demais regiões, o que indica melhor performance, apenas foram verificadas diferenças significativas para a performance no público feminino, onde aquelas residentes na região Sul apresentam melhor desempenho comparativamente a seus pares das demais regiões. Nesse sentido, para ambos os sexos, a região Sul foi considerada como categoria de referência para a análise realizada. Considerando estudos prévios que indicam uma relação inversa entre idade e desempenho em corredores de rua ${ }^{19,20}$, os resultados foram ajustados para essa variável.

Os resultados da análise de regressão confirmam a hipótese formulada, visto que corredores residentes na região Sul do país apresentam melhores desempenhos que aqueles oriundos das regiões Centro-oeste, Sudeste e Nordeste, para o sexo feminino, e Nordeste, para o público masculino. Tais resultados podem estar relacionados ao maior número de eventos existentes na referida região, o que cria uma atmosfera mais amigável para os corredores. Trabalho prévio, conduzido anteriormente pelo nosso grupo, com foco em uma abordagem multinível, indicou que o estado explica aproximadamente $3 \%$ da variância da performance entre corredores amadores brasileiros, de modo que a realização de eventos de atletismo foi a única variável a nível de estado que apresentou associação significativa com a performance ${ }^{21}$. Adicionalmente, o "Ranking das capitais brasileiras amigas da atividade física”, indicou que São Paulo, Belo Horizonte, Vitória, Curitiba e Rio de Janeiro são aquelas que apresentam os melhores indicadores ambientais (acesso a lazer, desenho urbano, estrutura viária para atividade física, trânsito e criminalidade) para prática de atividades físicas ao ar livre ${ }^{22}$.

Uma vez que a corrida de rua tende a ser realizada primordialmente em espaço outdoor, os praticantes estão expostos a um conjunto de restrições de ordem natural e física, como por exemplo aspectos climáticos (velocidade do vento, precipitação, umidade), e a existência de espaços adequados e seguros para o treinamento ${ }^{23,24}$. Desse modo, é possível que essas características estejam associadas ao envolvimento no treinamento (frequência, volume e duração), repercutindo no desempenho, especialmente entre o público feminino. Dadas as várias barreiras a que estão 
expostas (segurança pessoal, dupla jornada de trabalho, instalações de difícil acesso devido à segurança / violência urbana, cultura esportiva dominada por homens) ${ }^{25}$, diferentes estratégias têm sido delineadas para encorajar e disseminar a prática esportiva entre as mulheres $^{26}$. Portanto, dado o número de eventos e praticantes de corrida de rua na região Sul do país, é possível que a mesma apresente um ambiente mais acolhedor e seguro à prática da modalidade por parte das mulheres, o que pode explicar, em partes, o fato de as corredoras desta região apresentarem melhor desempenho, dado envolvimento com o treinamento, devido a um ambiente mais "amigável”.

Não obstante o uso alargado de questionários online ao longo dos últimos anos no meio acadêmico-científico ${ }^{27,28}$, bem como a validação prévia do instrumento utilizado para recolha de dados no presente estudo, sugerimos cautela na interpretação das informações. Na tentativa de reduzir os vieses, as informações referentes ao ritmo de corrida foram confirmadas, comparando-as aos resultados de provas oficiais, indicadas pelos participantes da pesquisa. Entretanto, reforçamos que o ritmo de corrida informado pelo participante não significa o melhor desempenho obtido pelo atleta, visto ser uma variável que se altera com o tempo; ou seja, os resultados podem ser alterados consoante o contexto/momento de análise. Adicionalmente, variáveis do sujeito e do contexto, que podem explicar esses resultados, não foram considerados no presente estudo, dado que foram exploradas em estudos prévios realizados pelo nosso $\operatorname{grupo}^{21,29,30}$. Por outro lado, destacamos a originalidade do trabalho, bem como a possibilidade de uso dessa informação para a organização de eventos da modalidade a nível nacional.

\section{CONCLUSÃO}

Em ambos os sexos, verificou-se que corredores de rua residentes na região Sul do país apresentam performance superior aos seus pares, sendo estas diferenças significativas entre as mulheres. Estudos futuros devem considerar a possibilidade de investigar diferenças de desempenho entre residentes de regiões/áreas urbanas e rurais, tendo em vista os espaços em que os treinamentos são realizados; bem como considerar o papel de fatores climáticos e econômicos das referidas regiões sobre as diferenças de desempenho dos corredores. Além disso, estudos futuros podem usar informações oriundas de eventos para a comparação de desempenho, bem como verificar se essas diferenças se mantém consoante prova de participação. 


\section{REFERÊNCIAS}

1. Andersen JJ. The state of running 2019: RunRepeat; 2019 [Available from: https://runrepeat.com/state-of-running.

2. IBGE IBdGeE-. Práticas de esporte e atividade física. Rio de Janeiro; 2015.

3. Thuany M, Gomes TN, Estevam LC, Almeida MBd. Crescimento do número de corridas de rua e perfil dos participantes no Brasil. Atividade física, esporte e saúde: temas emergentes. 1. Belém - PA: Rbf editora; 2021. 4. Ticket. Perfil do corredor brasileiro: https://blog.ticketagora.com.br/infografico-operfil-dos-corredores-2019/; 2019 [Available from:

https://blog.ticketagora.com.br/infografico-operfil-dos-corredores-2019/.

5. Danilo Balu. As 50 maiores corridas de rua do brasil (2017) Blog Recorrido: Blog Recorrido; 2017 [Available from: https://blogrecorrido.com/2018/01/15/as-50maiores-corridas-de-rua-do-brasil-2017/.

6. Thuany M, Knechtle B, Rosemann T, Almeida MBd, Gomes TN. Running around the Country: An Analysis of the Running Phenomenon among Brazilian Runners. Int J Environ Res Public Health. 2021;18(6610).

7. Breuer C, Hallmann K, Wicker P. Determinants of sport participation in different sports. Managing Leisure. 2013;16(4):269-86.

8. Galatti LR. Relatório Nacional de Desenvolvimento Humano do Brasil - AFEs, Desenvolvimento Humano e Esporte de Alto Rendimento: http://movimentoevida.org/; 2017 [

9. Tozetto AVB, Rosa RS, Mendes FG, Galatti LR, Souza ER, Collet C, et al. Local de nascimento e data de nascimento de medalhistas olímpicos brasileiros. Brazilian Journal of Kinanthropometry and Human Performance. 2017;19(3).

10. Costa ITd, Cardoso FdSL, Garganta J. O Índice de Desenvolvimento Humano e a data de nascimento podem condicionar a ascensão de jogadores de Futebol ao alto nível de rendimento? Motriz, Rio Claro. 2013;19(1):34-45.

11. Feizabadi MS, Khabiri M, Hamidi M. The relationship between the success of countries at the Guangzhou 2010 Summer Asian Games and demo-economic factors Procedia - Social and Behavioral Sciences. 2013;82:369 - 74 .

12. Reverdito RS, Galatti LR, Lima LA, Nicolau PS, Scaglia AJ, Paes RR. The "Programa Segundo Tempo" in Brazilian Municipalities: Outcome Indicators in Macrosystem. Journal of Physical Education. 2016;27(1).

13. Galatti LR. AFEs, Human Development and Sport High Performance. In R. N. d. D. H. d. Brasil (Ed.), Movement is life: physical and sports activities for all people. Brasília, DF: United Nations Development Program (UNDP). 2017.

14. InTrack Project. Welcome to InTrack Project 2021 [Available from: https://intrackproject.wixsite.com/website.

15. Thuany M, Gomes TN, Almeida MB. Validação de um instrumento para caracterização e verificação de fatores associados ao desempenho de corredores de rua. Scientia Plena. 2020;16(3):1-7.

16. Sebrae. Tendências do mercado de corrida de rua 2018 [Available from: https://sebraeinteligenciasetorial.com.br/prod utos/boletins-de-tendencia/tendencias-domercado-de-corridas-derua/5b5a1605d0a9751800f2af49.

17. Thuany M, Gomes TN, Souza RF, Almeida M. Onde estão os melhores corredores do Brasil? 2020.

18. Confederação Brasileira de Atletismo. Ranking Brasileiro 2020 http://www.cbat.org.br/novo/?pagina=ranking quadro\&t=b\&a=2020; 2020 [

19. Willy R, Paquette M. The physiology and biomechanics of the master runner. Sports Medicine and Arthroscopy Review. 2019;27(1):15-21.

20. Dahl J, Degens H, Hildebrand F, Ganse B. Do changes in middle-distance 
running kinematics contribute to the agerelated decline in performance? Journal of Musculoskeletal and Neuronal Interactions. 2020;20:94-100.

21. Thuany M, Gomes TN, Hill L, Rosemann TJ, Knechtle B, Almeida MBd. Running performance variability among runners from different brazilian states: A multilevel approach. International Journal of Environmental Research and Public Health. $2021 ; 18(7)$.

22. Hino AAF, Gonçalves PB, Reis RS.

Relatório do Ranking das Capitais Brasileiras Amigas da Atividade Física https://saude.abril.com.br/fitness/relatorio-doranking-das-capitais-brasileiras-amigas-daatividade-fisica/:

https://saude.abril.com.br/fitness/relatorio-doranking-das-capitais-brasileiras-amigas-daatividade-fisica/; 2018 [Available from: https://saude.abril.com.br/fitness/relatorio-doranking-das-capitais-brasileiras-amigas-daatividade-fisica/.

23. Allen-Collinson J. Running the routes together: co-running and knowledge in action. Journal of Contemporary Ethnography. 2008;37(1):38-61.

24. Ettema D. Runnable Cities. Environment and Behavior. 2016;48(9):112747.

25. Sportscotland. Barriers to women and girls' participation in sport and physical activity. 2008.

26. Running Sisters. Welcome to the Chilterns' Running Sisters 2019 [Available from: https://www.runningsisters.com/clubs.

27. Janssen M, Walravens R, Vos S, Thibaut E, Scheerder J, Brombacher A, et al. Understanding different types of recreational runners and how they use running-related technology. International Journal of Environmental Research and Public Health. 2020;17(7).

28. Andrew J Vickers, Vertosick EA. An empirical study of race times in recreational endurance runners. BMC Sports Science Medicine and Rehabilitation. 2016;8(1):26.
29. Thuany M, Gomes TN, Almeida MB. Is there any difference between "amateur" and "recreational" runners? A latent class analysis. Motriz, Rio Claro. 2020;26(4).

30. Thuany M, Gomes TN, Almeida MB. Fatores associados ao desempenho em corredores de rua. Revista Carioca de Educação Física. 2020;15(2):9.

Informação deste artigo/Information of this article:

Recebido: 27/06/2021

Aprovado: 03/11/2021

Publicado: 19/11/2021

Received: 27/06/2021

Approved: 03/11/2021

Published: 19/11/2021

Conflito de interesses/Conflicting Interests

Os autores declaram não ter conflitos de interesse.

The authors declare that they have no conflicting interests.

\section{Agradecimentos}

Agradecemos aos participantes da pesquisa. We thank the survey participants.

Como citar esse artigo / How to cite this article:

Thuany M, Gomes TN. Em qual região do Brasil, os corredores apresentam o melhor desempenho? Arq. Bras. Ed. Fís., Tocantinópolis, v. 4, n. 2, Ago./Dez. p. 132 141, 2021. 


\section{Tabelas}

Tabela 1. Informações descritivas [média (desvio padrão), frequência (\%)] da amostra estudada, consoante região de residência.

\begin{tabular}{|c|c|c|c|c|c|}
\hline Variáveis & $\begin{array}{c}\text { Centro-oeste } \\
(n=100)\end{array}$ & $\begin{array}{l}\text { Nordeste } \\
(n=420)\end{array}$ & $\begin{array}{l}\text { Norte } \\
(n=79)\end{array}$ & $\begin{array}{l}\text { Sudeste } \\
(n=4,22)\end{array}$ & $\begin{array}{c}\text { Sul } \\
(n=137)\end{array}$ \\
\hline \multicolumn{6}{|l|}{ Sexo } \\
\hline Feminino & $45(45 \%)$ & $153(36,4 \%)$ & $37(46,8 \%)$ & $164(38,9 \%)$ & $60(43,8 \%)$ \\
\hline Masculino & $55(55 \%)$ & $267(63,6 \%)$ & $42(53,2 \%)$ & $258(61,1 \%)$ & $77(56,2 \%)$ \\
\hline Idade (anos) & $39,5(9.9)$ & $37,5(9,9)$ & $35,7(10,4)$ & $38,8(8,9)$ & $36,7(8,5)$ \\
\hline IMC (kg.m²) & $24,1(3,3)$ & $24,3(3,1)$ & $24,6(3,9)$ & $24,6(3,3)$ & 23,9 $(3,2)$ \\
\hline Ritmo de corrida (seg/km) & $343,7(83,6)$ & $337,6(72,2)$ & $335,5(83,7)$ & $329,0(70,9)$ & $312,6(57,2)$ \\
\hline
\end{tabular}

Tabela 2. Resultados da regressão robusta para fatores associados ao desempenho em praticantes de corrida de rua do sexo feminino.

\begin{tabular}{cccc}
\hline Variáveis & Coeficiente & Erro padrão & Intervalo de confiança \\
\hline Constante & 271.39 & 17.7 & $236.4-306.2$ \\
Idade & 1.69 & 0.3 & $0.9-2.4$ \\
Centro-oeste & 49.11 & 14.6 & $20.3-77.8$ \\
Norte & 28.91 & 14.7 & $-0.1-57.9$ \\
Nordeste & 45.48 & 10.7 & $24.3-66.6$ \\
Sudeste & 29.45 & 10.8 & $8.1-50.7$ \\
\hline
\end{tabular}

Tabela 3. Resultados da regressão robusta para fatores associados ao desempenho em praticantes de corrida de rua do sexo masculino.

\begin{tabular}{cccc}
\hline Variáveis & Coeficiente & Erro padrão & Intervalo de confiança \\
\hline Constante & 228.4 & 10.0 & $208.6-248.2$ \\
Idade & 1.8 & 0.2 & $1.4-2.2$ \\
Centro-oeste & 8.2 & 10.1 & $-11.6-28.1$ \\
Norte & 10.1 & 10.7 & $-11.0-31.3$ \\
Nordeste & 17.1 & 7.2 & $2.9-31.4$ \\
Sudeste & 5.3 & 7.2 & $-8.8-19.6$ \\
\hline
\end{tabular}
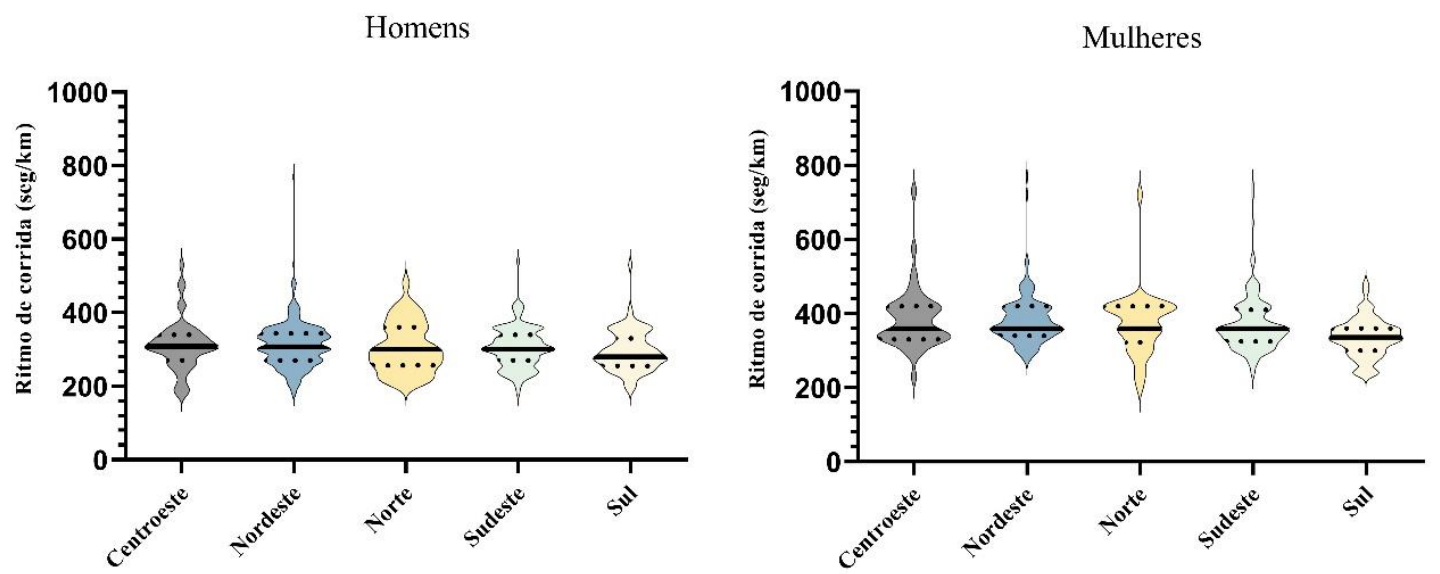

Figura 1. Mediana e range do desempenho, consoante região de residência e sexo ( ${ }^{*}$ diferenças significativas entre a região Sul demais regiões). 